\title{
Outcomes and predictors of recurrences after pulmonary vein isolation by cryoballoon or radiofrequency catheter atrial fibrillation ablation
}

\author{
Mihaela Grecu ${ }^{1}$, Mariana Floria ${ }^{*}, 2,3$, Elisabeta Hurjui ${ }^{1}$, Dana Mindru${ }^{1}$, Raluca Ozana \\ Chistol $^{1}$, Catalina Arsenescu Georgescu ${ }^{1,2}$, Grigore Tinica ${ }^{1,2}$
}

${ }^{1}$ Cardiovascular Disease Institute lasi, Romania; ${ }^{2 " G r i g o r e ~ T . ~ P o p a " ~ U n i v e r s i t y ~ o f ~ M e d i c i n e ~ a n d ~}$ Pharmacy lasi, Romania; “'Sf. Spiridon” Clinical County Emergency Hospital, lasi, Romania

\begin{abstract}
Aim: Catheter-based pulmonary vein isolation (PVI) has become a new option treatment for drug-resistant and symptomatic paroxysmal atrial fibrillation (AF). We aimed to assess the outcomes after radiofrequency and cryoballoon catheter AF ablation. Material and methods: We performed a single-center prospective clinical study enrolling patients who underwent catheter ablation for paroxysmal AF between 2013 and 2015. The main objective was the outcomes after AF ablation by cryoballoon or radiofrequency energy. Follow-up was performed at 3, 6 and 12 months after ablation. Results: A total of 85 patients were included: 64 assigned to radiofrequency and 21 to cryoballoon ablation. Overall success rate at 3,6 and 12 months post-ablation was $62 \%, 76 \%$ and $75 \%$, respectively. Redo procedure was required in 8 patients $(10.3 \%)$ at 6 months. Success rate at 12 months was non-significantly higher in radiofrequency ablation group ( $76 \%$ versus $62 \% ; p=0.56)$. Complication rates were equivalent, without life treating or permanent sequels. Men gender, AF duration, obesity and sleep apnoea syndrome were clinical predictors of AF recurrence at 12 months after procedure. Conclusions: Cryoballoon and radiofrequency ablation appear to be safe and effective procedures for $A F$ treatment, with similar success rate. Men gender, AF duration, obesity and sleep apnea syndrome seem to be associated with AF recurrence after ablation.
\end{abstract}

Keywords: atrial fibrillation, catheter ablation, cryoballoon, radiofrequency, pulmonary vein isolation

\section{Introduction}

Atrial fibrillation $(\mathrm{AF})$ is the most common sustained cardiac arrhythmia associated with important morbidity and mortality. Catheterbased pulmonary vein isolation (PVI) has become a standard treatment for drugresistant and symptomatic paroxysmal $\mathrm{AF}$ [1].

Received: August 2017; Accepted after review: September 2017; Published: September 2017.

*Corresponding author: Mariana Floria, MD, PhD, Emergency Hospital and "Grigore T. Popa" University of Medicine and Pharmacy, No. 16, Universitatii Street, 700115, lasi, Romania.

E-mail: floria mariana@yahoo.com
The increasing number of patients with drug refractory $A F$, as well as electrophysiology laboratories, will create in the next years an economic issue, which every cardiac electrophysiologist will have to face with [2]. Identification of AF recurrence predictors after catheter ablation is essential to reduce health costs and improve long-term results of this intervention.

Open irrigated radiofrequency (RF) and cryoballoon catheter ablation are the main techniques used for $\mathrm{PVI}$ as new treatment option in AF. In the last years, the cryoballoon based technique is used as a promising alternative with a shorter learning curve. The lack of data on the comparative efficacy and procedural safety between these two 
techniques has encouraged different studies in recent years. In this single-center prospective clinical study we assessed the outcomes after catheter ablation using cryoballoon versus RF in patients with paroxysmal AF.

\section{Material and methods}

In this single center clinical prospective study, patients with paroxysmal AF referred to undergo PVI, between 2013 and 2015, were included. The ablation procedure consisted of endocardial isolation of each PV antrum by RF energy or cryoablation.

\section{Patient population}

We enrolled prospectively and consecutively patients with symptomatic refractory paroxysmal AF referred for endocardial catheter ablation. Paroxysmal AF was defined as $\mathrm{AF}$ that terminates spontaneously or with intervention within 7 days of onset [2]. Patients with continuous AF who undergo cardioversion within seven days were classified as having paroxysmal $\mathrm{AF}$ if the cardioversion was performed within 48 hours of $\mathrm{AF}$ onset, and persistent $\mathrm{AF}$ if the cardioversion was performed more than 48 hours after AF onset [2].

Patient's selection and inclusion in the study was made according with the current guidelines [1, 2]. Inclusion criteria were: symptomatic patients aged more than 18 years and documented refractory paroxysmal AF referred for endocardial catheter ablation. Patients were fully informed about the nature of the study and provided written informed consent which was approved by the local Ethics Committee.

Exclusion criteria were: patients with valvular AF, patients with advanced left ventricular systolic function impairment (ejection fraction<35\%), patients with significant chronic obstructive pulmonary disease (peak expiratory flow $<40 \%$ ), comorbidity with reduced life expectancy, anticipated protocol non-compliance that would limit follow-up, presence of left atrial or left atrial appendage thrombus (detected by bidimensional transesophageal echocardio- graphy), pregnant women or planned to become pregnant during the study, and patients who refused to sign informed consent.

\section{Endocardial catheter ablation procedure}

The ablation procedure consisted of endocardial antral isolation of each pulmonary vein antrum by radiofrequency energy using a Lasso $^{\mathrm{TM}}$ catheter (BiosenseWebster, CA, USA) and an open-irrigated non-force sensing catheter tip Celsius ${ }^{\mathrm{TM}}$ Thermo Cool (Biosense Webster, CA, USA) or cryo-ablation (ICE ${ }^{\circledR}$ Cryoablation System, Medtronic, USA) using the first-generation cryoballoon, as currently recommended 1,2. Both procedures were done using local anesthesia by femoral access and deep sedation with Morphine fractionated intravenous during application of energy. Antral PVI was obtained at $35 \mathrm{~W}$ (by RF energy) and minus $40^{\circ}$ (by cryotherapy). In patients with paroxysmal AF longer than 24 hours we used exclusively RF energy and ablation procedure was completed by complex fractionated atrial electrogram and cavotricuspid istmus ablation (if common atrial flutter was confirmed before or during procedure).

\section{Pre- and Post-ablation Care and Follow- up}

Before the procedure, a 12 leads ECG recording, polysomnography, transthoracic and transesophageal echocardiography and computer-tomography (CT) of the pulmonary veins were performed in all patients. Antiarrhythmic medications except amiodarone was stopped more than five halflives prior to ablation. All patients were taking acenocumarol during a minimum 4-8 weeks before intervention. Documented INR (International Normalized Ratio) between 2 and 3 was mandatory for at least 3 times prior to the procedure. Acenocumarol was continued during the procedure for an INR value about 2. Transesophageal echocardiography was performed 24 hours before intervention and during the procedure. An intracardiac thrombus was ruled out in all patients. 
After the procedure the patients were clinically monitored during in-hospital stay for 2 or 3 days.

The follow-up planned at 3,6 and 12 months consisted in: clinical examination, 12 leads ECG, 24 hours Holter monitoring, and transthoracic echocardiography (for indexed left atrial volume and left ventricular ejection fraction assessed by Simpson biplane formula).

Adverse events were evaluated during hospitalization, at every follow-up and anytime during the study by the cardiologist. Antiarrhythmic and anticoagulation drugs were continued in all patients until the first follow-up. Success rate was defined as absence of AF recurrence, without antiarrhythmic drugs, confirmed by 24 hours ECG Holter monitoring. A redo procedure was decided at 6 months depending on AF burden revealed by 24 hours ECG Holter monitoring.

The primary end point in our study was the success rate at 12 months after AF ablation. The secondary end point were mean procedural time, mean energy application time, X-ray exposure, redo procedure and complication rate at 6 months in the RF group versus cryoballoon group. Parameters associated with AF recurrences after procedure were also assessed.

\section{Statistical analysis}

Data are presented as frequency distributions and simple percentages. Continuous variables are expressed as mean \pm standard deviation. Multivariable analysis was used to identify the parameters associated with AF recurrences post-ablation procedure. Statistical analysis was performed using SPSS 11.5 for Windows (SPSS Inc., Chicago, IL, USA). A $\mathrm{p}$ value (from paired sample $T$ test) $\leq 0.05$ was considered significant.

\section{Results}

A total of 85 patients $(51 \%$ men; mean body mass index of $28 \pm 3.4 \mathrm{~kg} / \mathrm{m}^{2}$; mean age of $57.4 \pm 8$ years; mean AF duration of $6 \pm 4.5$ years) were included. Clinical baseline characteristics of the patients at baseline are shown in Table 1. The majority of patients were hypertensive and with sleep apnea syndrome. Thromboembolic risk was predominantly intermediate.

Table 1. Clinical baseline characteristics of patients included in the study $(n=85)$. CHADS2 and CHA2DS2VASC: thromboembolic risk scores.

\begin{tabular}{cc}
\hline Parameter & Results \\
\hline Hypertension (\%) & $40(47)$ \\
\hline Diabetes mellitus (\%) & $12(14)$ \\
\hline $\begin{array}{c}\text { Cardiomyopathy (\%) } \\
\text { Prior stroke or transient } \\
\text { ischemic attack (\%) }\end{array}$ & $12(14)$ \\
\hline $\begin{array}{c}\text { Lone atrial fibrillation (\%) } \\
\text { CHADS2 (mean value } \pm \text { SD) }\end{array}$ & $17(20)$ \\
\hline CHA2DS2VASC (mean value & $0.8 \pm 0.7$ \\
\hline ISD) & $1.2 \pm 0.9$ \\
\hline Apnea-hypopnea index $\geq 10$ & $48(56 \%)$ \\
\hline
\end{tabular}

Sixty four out of these patients $(75 \%)$ underwent a first time RF catheter ablation and 21 patients $(25 \%)$ underwent cryoballoon catheter ablation. The intraprocedural and postprocedural catheter ablation results are shown in Table 2. The RF group required significantly longer procedural and energy application time, while the cryoballoon group registered a significantly higher $\mathrm{X}$-ray (fluoroscopy) exposure. Success rate at 12 months was non-significantly higher in RF ablation group. Complication rate at 6 months was similar in the two groups. There was one cardiac tamponade and one ischemic stroke in 
RF ablation group and one femoral fistula and one phrenic nerve palsy in cryoablation group. The last one complication was reversible under rehabilitation in 12 months. No major (fatal) complications or symptomatic pulmonary vein stenosis were diagnosed. Redo procedure was significantly more frequently necessary in cryoablation group.

Table 2. Comparative intraprocedural and postprocedural catheter ablation results. RF: radiofrequency; Cryo: cryoablation.

\begin{tabular}{lccc}
\hline \multicolumn{1}{c}{ Parameter } & $\begin{array}{c}\text { RF } \\
\text { N=64 }\end{array}$ & $\begin{array}{c}\text { CRYO } \\
\mathbf{N = 2 1}\end{array}$ & P \\
\hline Mean procedural time (min) & $310 \pm 80$ & $215 \pm 27$ & 0.037 \\
$\begin{array}{l}\text { Mean energy application time } \\
\text { (min) }\end{array}$ & $42 \pm 21$ & $40 \pm 7$ & 0.043 \\
X-ray exposure (min) & $27 \pm 14$ & $34 \pm 7$ & 0.017 \\
\hline Complications (\%) & $2(3.1)$ & $2(9.5)$ & 0.73 \\
\hline Success at 12 months (\%) & $45(76)$ & $13(62)$ & 0.56 \\
\hline Redo procedure (\%) & $5(7.8)$ & $3(14)$ & 0.047 \\
\hline
\end{tabular}

A total of $100 \%, 98 \%$ and $85 \%$ of scheduled follow-up visits were attended at 3 , 6 and 12 months, respectively. The presence of sinus rhythm was confirmed in $62 \%, 76 \%$ and $75 \%$ of patients at the scheduled follow-up visits (Figure 1). Redo procedure was performed in 8 patients $(10.3 \%)$ at 6 months follow-up. A total of $10.3 \%$ of patients underwent a redo procedure after pulmonary vein reconnection at 6 months, while $23.1 \%$ of patients required antiarrhythmic drugs.

Significantly reverse left atrial remodeling assessed by indexed left atrial volume was obtained (from $48.9 \pm 23.4 \mathrm{ml}$ before procedure to $43.6 \pm 17.1 \mathrm{ml}$ at 12 months; $\mathrm{p}=0.026$ ) due to a stable sinus rhythm after PVI isolation.

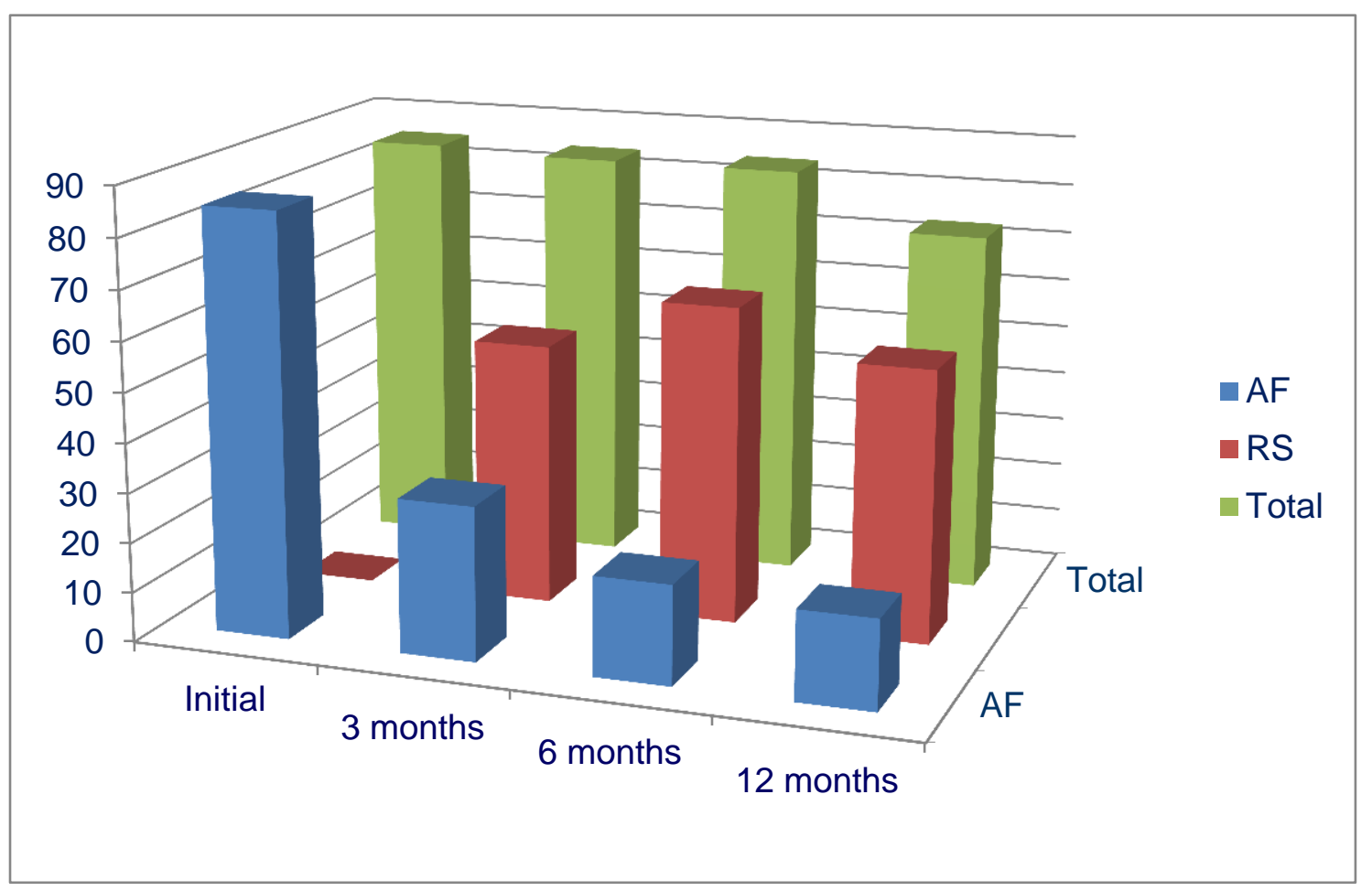

Fig. 1. Overall freedom from atrial fibrillation at 3,6 and 12 months. AF: atrial fibrillation; RS: sinus rhythm. 
Comparative data for patients with/without AF recurrence at 12 months are presented in Table 3. A total of $15 \%$ of patients were lost from follow-up. Significantly more men were in sinus rhythm at 12 months. Men gender, AF history, obesity and sleep apnea syndrome were identified by multivariable analysis with $69 \%$ sensibility and $69.8 \%$ specificity as clinical predictors of AF recurrence after PVI.

Table 3. Comparative data for patients with/without atrial fibrillation recurrence at 12 months. AF: atrial fibrillation; AHI: apnea hypopnea index; BMI: body mass index; PAF: paroxysmal atrial fibrillation.

\begin{tabular}{|c|c|c|c|}
\hline Clinical parameters & $\begin{array}{c}\text { Sinus rhythm group } \\
\text { (53 pts) }\end{array}$ & $\begin{array}{l}\text { Recurrence group } \\
\text { (10 pts) }\end{array}$ & $\mathbf{P}$ \\
\hline Men (\%) & $39(73.6)$ & $4(40.0)$ & $0.046^{a}$ ) \\
\hline PAF less 24 h (\%) & $41(77.4)$ & $8(80)$ & 0.610 \\
\hline PAF less than 7 days (\%) & $12(22.6)$ & $2(20.0)$ & $\left.0.610^{a}\right)$ \\
\hline AF history (years) & $5.24 \pm 4.21$ & $6.83 \pm 5.35$ & $0.315^{b)}$ \\
\hline Obesity (BMI $\geq 30 \mathrm{~kg} / \mathrm{m} 2)$ & $20(37.7 \%)$ & $4(40.0 \%)$ & 0.579 a) \\
\hline Sleep apnea $(A H I \geq 10)$ & $31(59.6 \%)$ & $6(60.0 \%)$ & 0.634 a) \\
\hline $\begin{array}{l}\text { Indexed left atrial volume } \\
\left(\mathrm{ml} / \mathrm{m}^{2}\right)\end{array}$ & $43.62 \pm 18.52$ & $42.54 \pm 9.41$ & $0.874^{b)}$ \\
\hline $\begin{array}{l}\text { Left ventricular ejection fraction } \\
<50 \%\end{array}$ & $5(9.4 \%)$ & $0(0 \%)$ & $\left.0.408^{a}\right)$ \\
\hline
\end{tabular}

a) $p$ values with Chi square $\quad$ b) $p$ values with Test $F$ (ANOVA)

\section{Discussions}

The 2016 ESC Clinical Practice Guideline on the management of $A F$ has stated the central role of catheter ablation in the treatment of drug-refractory patients who have symptomatic recurrences of AF [2]. Many of the focal electrical activities that initiate and sustain AF have been found to reside within or near the pulmonary veins. Consequently, PVI by catheter ablation has emerged as the standard approach to eliminate the focal triggers associated with $\mathrm{AF}[1,2]$.

The most common method is the use of RF current applied in a point-by-point mode, in order to produce tissue heating and cellular necrosis. Cryoballoon ablation of the pulmonary veins is a new technique expected to have similar results to RF procedure, but with fewer complications. The method uses cryogenic energy applied with a balloon in a single-step mode, which leads to necrosis by freezing. Even if it involves a higher radioscopy exposure, the cryoballoon seems more appealing because it can be practiced by conventional means, creating a circular lesion around each pulmonary vein in a relatively simple manner. In opposition, RF ablation is a complex procedure that requires extensive training, since the cardiac anatomy is reconstructed with all its peculiarities with the use of an electro-anatomical mapping system (Carto 3D).

Radiofrequency ablation has the advantage of being applicable regardless of the mechanism of AF initiation (pulmonary vein triggers, non-pulmonary vein triggers, micro or macro-reentry) or perpetuation, while cryoballoon ablation can be applied in AF initiation mechanism exclusively by pulmonary vein ectopies. Also, in choosing the appropriate treatment method, one must consider that cryoballoon ablation is indicated only in patients with paroxysmal AF and structurally normal heart, whereas RF ablation can be applied in patients with paroxysmal or persistent $\mathrm{AF}$ and an $\mathrm{AF}$ substrate (cardiomyopathy).

Pulmonary-vein isolation is a challenging ablation strategy in the treatment of patients with paroxysmal or persistent AF. Achieving acute pulmonary vein isolation does not guarantee long-term electrical isolation of the pulmonary veins. Success rate is affected by patient characteristics because AF includes different subtypes and occurs in different clinical subsets. Identifying the predictors of sinus rhythm maintenance after catheter 
ablation is a highly desirable objective since it would help the community of cardiac electrophysiologists in reducing unnecessary procedures, in limiting complications and in reducing health care costs. We assessed the outcomes after these two techniques, and we identified the parameters associated with AF recurrence at 12 months after $\mathrm{PVI}$.

Previous studies comparing RF ablation with cryoballoon ablation have found comparable results of these two technologies [3-7]. Only a small number of studies showed a higher efficacy of cryoballoon ablation [8-10] and a trend for more recurrences and complications in the RF group (probable due to more favorable risk profile in patients undergoing cryoballoon ablation) [11]. In this study, RF ablation has had similar results as cryoballoon ablation at 12 months. Cryotherapy was associated with shorter ablation time, but higher fluoroscopy time. Overall complication rate was $5.1 \%$ in our study, lower than the $13.5 \%$ rate reported in the Sustained Treatment of paroxysmal Atrial Fibrillation (STOP AF) trial [12]. However a large recently published metaanalysis shown that cryoballoon ablation seems to be associated with greater freedom from $A F$, shorter procedural time, and lower rate of major complications, compared with radiofrequency ablation [13].

We found a significant reverse left atrial remodeling after $\mathrm{PVI}$ due to maintenance of stable sinus rhythm. The benefits of AF ablation and long term success seem to be dependent on the stage of LA structural remodeling and not on paroxysmal or persistent nature of AF [14]. The reverse remodeling of the LA structure and function after catheter ablation of AF is significantly better when performed in the early stage of arrhythmia with mild LA structural remodeling [14].

Using multivariable analysis we found that men gender, AF history, obesity and sleep apnea syndrome were clinical predictors of $\mathrm{AF}$ recurrence with a good sensibility and specificity. However, identification of AF recurrence predictors after catheter ablation is a challenging task due to the extreme heterogeneity of the data published in the literature [15-17].
Although, aggressive risk factor management improved the long-term success of AF ablation [18], only aggressive blood pressure treatment did not reduce atrial arrhythmia recurrence after catheter ablation for AF [19]. Therefore it is very difficult to analyze taking into consideration all factors associated with success rate or recurrences after AF ablation, irrespective of technique used for PVI.

\section{Study limitations}

This is a small prospective nonrandomized study. All patients included in the study were with paroxysmal AF less than 24 hours or less than 7 days. Therefore, it might be discussed about a kind of inhomogeneous study group even all patients according with current definition were with paroxysmal AF. The small number of patients included in the study made more difficult interpretation of the results in the subgroups study. For example, it cannot be excluded the contribution of obesity or sleep apnea or AF duration when analyzing comparative data in patients with recurrences versus success rate.

\section{Conclusion}

Cryoballoon and radiofrequency ablation appear to be safe and effective procedures for atrial fibrillation treatment, with similar success rate. Men gender, atrial fibrillation duration, obesity and sleep apnea syndrome seem to be associated with atrial fibrillation recurrence after ablation.

\section{Conflict of interest}

The authors declare that they have no competing interests.

\section{Funding}

This work was supported by the Project entitled: Expanding and upgrading an Atrial Fibrillation Treatment Research Center as a method of preventing heart failure by developing the research and development infrastructure. 


\section{References}

1. $2012 \mathrm{HRS} / \mathrm{EHRA} / \mathrm{ECAS}$ Expert Consensus Statement on Catheter and Surgical Ablation of Atrial Fibrillation: Recommendations for Patient Selection, Procedural Techniques, Patient Management and Follow-up, Definitions, Endpoints, and Research Trial Design: A report of the Heart Rhythm Society (HRS) Task Force on Catheter and Surgical Ablation of Atrial Fibrillation. Europace 2012; 14:528-606.

2. Kirchhof P, Benussi S, Kotecha D, et al. 2016 ESC Guidelines for the management of atrial fibrillation developed in collaboration with EACTS. Eur Heart J 2016; 37(38):2893-2962.

3. Kuck $\mathrm{KH}$, Brugada J, Fürnkranz $\mathrm{A}$, et al. Cryoballoon or radiofrequency ablation for paroxysmal atrial fibrillation. $N$ Engl $J$ Med 2016; 374:2235-2245.

4. Luik A, Radzewitz A, Kieser $M$, et al. Cryoballoon versus open irrigated radiofrequency ablation in patients with paroxysmal atrial fibrillation: the prospective, randomized, controlled, noninferiority Freeze AF Study. Circulation 2015; 132:1311-1319.

5. Wasserlauf J, Pelchovitz DJ, Rhyner J, et al. Cryoballoon versus radiofrequency catheter ablation for paroxysmal atrial fibrillation. Pacing Clin Electrophysiol 2015; 38:83-89.

6. Jourda F, Providencia R, Marijon E, et al. Contact-force guided radiofrequency vs. second-generation balloon cryotherapy for pulmonary vein isolation in patients with paroxysmal atrial fibrillation-a prospective evaluation. Europace 2015; 17:225-231.

7. Squara F, Zhao A, Marijon E, et al. Comparison between radiofrequency with contact forcesensing and second-generation cryoballoon for paroxysmal atrial fibrillation catheter ablation: a multicenter European evaluation. Europace 2015; 17:718-724.

8. Aryana A, Singh SM, Kowalski M, et al. Acute and long-term outcomes of catheter ablation of atrial fibrillation using the second-generation cryoballoon versus open irrigated radiofrequency: a multicenter experience. $J$ Cardiovasc Electrophysiol 2015; 26:832-839.

9. Hunter RJ, Baker V, Finlay MC, et al. Point-bypoint radiofrequency ablation versus the cryoballoon or a novel combined approach: a randomized trial comparing 3 methods of pulmonary vein isolation for paroxysmal atrial fibrillation (the Cryo Versus RF Trial). J Cardiovasc Electrophysiol 2015; 26:1307-1314.

10. Straube F, Dorwarth U, Ammar-Busch S, et al. First-line catheter ablation of paroxysmal atrial fibrillation: outcome of radiofrequency vs. cryoballoon pulmonary vein isolation. Europace 2016; 18(3):368-375.

11. Packer DL, Kowal RC, Wheelan KR, et al. Cryoballoon ablation of pulmonary veins for paroxysmal atrial fibrillation: first results of the North American Arctic Front (STOP AF) pivotal trial. J Am Coll Cardiol 2013; 61:1713-1723.

12. Liu $X H$, Chen $C F$, Gao $X F, X u Y Z$. Safety and efficacy of different catheter ablations for atrial fibrillation: a systematic review and metaanalysis. Pacing Clin Electrophysiol 2016; 39(8):883-899.

13. Kuppahally SS, Akoum N, Burgon NS, et al. Left atrial strain and strain rate in patients with paroxysmal and persistent atrial fibrillation: relationship to left atrial structural remodeling detected by delayed-enhancement MRI. Circ Cardiovasc Imaging 2010; 3:231-239.

14. Hsieh MH, Tai CT, Tsai CF, et al. Clinical outcome of very late recurrence of atrial fibrillation after catheter ablation of paroxysmal atrial fibrillation. J Cardiovasc Electrophysiol 2003; 14(6):598-601.

15. Mainigi SK, Sauer WH, Cooper JM, et al. Incidence and predictors of very late recurrence of atrial fibrillation after ablation. $J$ Cardiovasc Electrophysiol 2007; 18(1):69-74.

16. Weerasooriya $R$, Khairy $P$, Litalien $J$, et al. Catheter ablation for atrial fibrillation: are results maintained at 5 years of follow-up? $J$ Am Coll Cardiol 2011; 57(2):160-166.

17. Pathak RK, Middeldorp ME, Lau DH, et al. Aggressive risk factor reduction study for atrial fibrillation and implications for the outcome of ablation: the ARREST-AF cohort study. $J \mathrm{Am}$ Coll Cardiol 2014; 64(21):2222-2231.

18. Parkash R, Wells GA, Sapp JL, et al. Effect of aggressive blood pressure control on the recurrence of atrial fibrillation after catheter ablation: a randomized, open-label clinical trial (SMAC-AF [substrate modification with aggressive blood pressure control]). Circulation 2017; 135(19):1788-1798. 